\title{
A Pilot Study of Concentrated EMDR: A Brief Report
}

\author{
Earl Grey \\ Walden University, Chrysalis Mental Health \& Wellness Inc., Pittsburgh, Pennsylvania
}

\begin{abstract}
The current research used a quantitative single-case study design to investigate the effectiveness of eye movement desensitization and reprocessing (EMDR) treatment for a participant diagnosed with comorbid major depressive disorder (MDD), severe without psychotic features, and panic disorder with agoraphobia. Treatment frequency was three sessions per week, with twelve 90-minute reprocessing sessions provided over a period of 1 month; the study also evaluated this application of "concentrated EMDR." At baseline, mean scores on the Beck Depression Inventory-II (BDI-II) and Beck Anxiety Inventory (BAI) were 49 and 38 , and at 3-month follow-up, the scores had decreased to 8 and 7 respectively. The results of this pilot study indicate that concentrated EMDR may be effective in treating comorbid MDD and panic disorder with agoraphobia. The study also evaluated the application of concentrated EMDR, with treatment frequency increased from one session to three sessions per week. Twelve 90-minute reprocessing sessions were provided over a period of 1 month. Results show the apparent effectiveness of concentrated EMDR.
\end{abstract}

Keywords: EMDR; depression; panic; concentrated EMDR; evidence-based practice

$\mathbf{E}$ ye movement desensitization and reprocessing (EMDR) is a psychotherapy approach that was originally developed for the treatment of traumatic memories (Shapiro, 1989). Although its application has since been expanded to address a range of disorders and problems (Shapiro, 2001), most of the clinical research has investigated its effectiveness in the treatment of posttraumatic stress disorder (PTSD). Multiple randomized clinical trials have established its efficacy, and EMDR is now recognized internationally as an evidence-based treatment for PTSD. For example, it was designated a category " $A$ " treatment for trauma by the U.S. Department of Veterans Affairs (VA) and U.S. Department of Defense (DoD; 2004) and the American Psychiatric Association (APA; 2004). The U.K. National Institute for Clinical Excellence (2005) have also identified EMDR as an empirically supported treatment for PTSD.

\section{Eye Movement Desensitization and Reprocessing}

EMDR is theoretically grounded in Shapiro's (2001) adaptive information processing (AIP) model. This model posits that dysfunctionally stored memories are the basis of clinical pathology (Solomon \& Shapiro,
2008). Shapiro theorizes that when a disturbing life event is inadequately processed, it is manifested with somatic, affective, cognitive, and perceptual symptoms. The cognitive components are understood to manifest as negative self-evaluations. When the disturbing unprocessed event occurs in childhood, such conclusions of self (Grey, 2010) are evident across an individual's lifetime function in maladaptive themes of being overly responsible, unsafe, powerless, and/or valueless (Stewart-Grey, 2008). From an AIP perspective, these maladaptive themes are viewed as components of unprocessed memories, which now interfere with current life function, blocking the individual from attaining their potential and achieving their life goals.

The AIP model theorizes that EMDR processing of the disturbing memory will result in transformation of maladaptive themes and comprehensive symptom resolution. EMDR treatment uses a standardized three-pronged protocol, which accesses and processes (a) memories of the disturbing past events, (b) current situations that trigger the disturbance, and (c) imaginal templates for appropriate future action (Shapiro, 2001). EMDR uses an eight-phase protocol.

EMDR commences with history taking (Phase 1) and client preparation (Phase 2). The EMDR clinician and client work together to identify targets for EMDR 
processing, in addition to standard history-taking procedures. Potential targets include unprocessed memories of traumatic events (designated as large " $T$ " trauma by Shapiro, 2001) and other disturbing life events (small " $t$ " trauma), as well as current situations, which trigger distress and future desired outcomes. During Phases 1 and 2, the clinician assesses the client's stability, his or her ability to regulate affect and to manage potential spikes in symptoms between sessions (Shapiro, 2001). If necessary, specific skills are taught and/or various procedures are used to assist the client in developing sufficient stability to engage in reprocessing.

Once the client has achieved a suitable level of stability and affects regulation, the clinician progresses into Phases 3-8 of the protocol. Phases 3-7 are the reprocessing phases directed at suitable targets as delineated in the three-pronged protocol. In Phase 3, the memory and its cognitive, affective, and sensory elements are accessed. The client identifies a related image, emotions, body sensations, a self-referencing current negative cognition (NC; e.g., "I'm valueless"), and a preferred positive cognition (PC; e.g., "I have value"). The client provides ratings of distress using the Subjective Units of Disturbance (SUD) scale (Shapiro, 2001; Wolpe, 1990), where $0=$ no disturbance and $10=$ worst possible disturbance. The client also rates the validity of the desired PC using the Validity of Cognition (VOC) scale (Shapiro, 2001), where $1=$ untrue and $7=$ completely true. Phase 4 desensitizes the distress, reducing the SUD rating to 0 . Phases 5 and 6 eliminate residual dysfunctional material, strengthen additional adaptive networks, and establish the PC, increasing the VOC rating to 7 . Phase 7 brings the client to equilibrium and provides guidance to manage symptoms in-between treatment sessions. Phase 8 is conducted at the beginning of each treatment session and reevaluates the previous sessions' work and the overall treatment plan.

\section{Eye Movement Desensitization and Reprocessing Treatment Frequency}

Historically, mental health sessions seem to be scheduled on a weekly basis, unless otherwise clinically indicated. The weekly session schedule seems to be used and accepted, not out of scholarly knowledge, but by tradition. The weekly treatment schedule is an element of treatment provision that should be scientifically examined.

EMDR has usually been provided on this onesession-per-week basis. The research studies, which evaluated EMDR's efficacy in the treatment of PTSD, have typically used a one-session-per-week schedule. Indirectly, these studies have supported treatment application at that level of frequency, but this frequency has never been directly tested. There are some case studies in which acute stress has been treated with EMDR or other early EMDR interventions (Shapiro \& Laub, 2008). For example, Wesson and Gould (2009) reported on the successful application of EMDR over 4 consecutive days for a soldier with acute stress reaction. This application of concentrated EMDR produced a positive outcome, with the soldier becoming asymptomatic and returning immediately to frontline duties. His treatment response was reflected in large changes on four standardized measures with results maintained at 18-month follow-up. The current study evaluates the application of concentrated EMDR, with a treatment schedule at the rate of three weekly sessions.

\section{EMDR Treatment of Depression}

There are few studies of EMDR treatment of major depressive disorder (MDD). Bae, Kim, and Park (2008) conducted a case study of EMDR treatment of two female adolescents diagnosed with MDD. The first participant was a 16-year-old who became depressed about a year after her father died of liver disease. She received three EMDR sessions, which focused on various memories of her father: his life and his death. The second participant was a 14-year-old who became depressed about a year after her father had an extramarital affair and abandoned the family. She received seven EMDR sessions, which focused on her relationship with her father and her interactions with peers. Bae et al. (2008) found that in both cases, EMDR eliminated depressive symptoms, with full remission of the diagnosis of MDD. These cases are noteworthy because the memories addressed with EMDR were about disturbing life events rather than traumatic events.

More recently, Uribe, Ramírez, and Mena (2010) evaluated the effects of a mean of 12 EMDR sessions on the cognitive processing of 3 participants diagnosed with MDD. They tested the hypothesis that EMDR treatment would resolve the beliefs, emotions, and sensations related to the processed memory, and that related cognitions would become more adaptive. Uribe et al. (2010) found that EMDR produced changes at an "unconscious level in the bias mechanisms in the negative valence evaluation." They further found a positive treatment effect on participants' emotional organization and the structure of negative information. These results support the positive impact EMDR can have on the cognitive and emotional architecture in depressed clients. 
EMDR has been found to alleviate depressive symptoms. Broad and Wheeler (2006) used a case study design to investigate the effect of EMDR with an adult who reported depressive symptoms. Through traditional EMDR treatment, which addressed early childhood medical trauma, the symptoms of depression were alleviated and the use of an antidepressant was eliminated. Although very limited, the positive results of these investigations of EMDR and MDD provide a scholarly need to further investigate EMDR's impact on depression.

When investigating the effects of EMDR for the treatment of PTSD, researchers have also assessed the impact of EMDR on concomitant symptoms of depression and other anxiety spectrum disorders, finding that such symptoms showed improvements with EMDR. For example, in their study of EMDR treatment of female survivors of childhood sexual abuse, Edmond, Rubin, and Wambach (1999) determined that EMDR produced statistically significant improvements in depression and anxiety. Similarly, Power et al. (2002) compared EMDR treatment and exposure plus cognitive restructuring for participants diagnosed with PTSD. They found that both treatments produced similar significant pre/ post symptom reduction and that EMDR was superior on client self-report measures of depression and social functioning. In Högberg et al.'s (2007) study on chronic PTSD treatment, participants receiving EMDR showed significant posttreatment changes on the Hamilton Rating Scale for Depression (HAM-D). Similarly, in their randomized comparison of EMDR and fluoxetine, van der Kolk et al. (2007) reported that not only EMDR improved the symptoms of PTSD and depression, but also the EMDR group continued to gain benefits from EMDR after the treatment had ceased.

\section{EMDR Treatment of Anxiety Disorders}

Because PTSD is an anxiety spectrum disorder, there has been some investigation of treating anxiety spectrum disorders with EMDR, although limited only. In their review of the research on EMDR and anxiety spectrum disorder, de Jongh and ten Broeke (2009) stated that "EMDR's efficacy for other conditions than PTSD is still scarce" (p. 137). Several studies have specifically examined EMDR treatment of panic disorder and specific phobia. Although EMDR proved more efficacious than no treatment, it seems that cognitive behavioral therapy (CBT) and exposure therapy yielded more positive results. Feske and Goldstein's (1997) controlled outcome study evaluated the treatment of panic disorder, comparing EMDR to a non-eye movement variant and wait list. They found that EMDR appeared superior at posttreatment, but that there was no difference between treatments at follow-up. A study by Goldstein, de Beurs, Chambless, and Wilson (2000) compared EMDR to association and relaxation therapy (ART) to treat panic disorder with agoraphobia. Although their results indicated that EMDR produced superior results when compared to the wait list group, it was not any better than ART in reducing panic severity, agoraphobia, or anxiety. In 2007, Fernandez and Faretta highlighted the importance of client preparation and therapist fidelity to the EMDR treatment protocols. In their single case study of EMDR treatment of panic and agoraphobia, they designated more time for history taking and preparation (six sessions) than Goldstein et al. (2000; one session) did. Fernandez and Faretta reported a positive effect, with remission of panic and agoraphobic symptoms and with results maintained through 1-year follow-up.

De Jongh and ten Broeke (2009) also argued that some of the anxiety spectrum studies had poor methodology and that the outcomes may not be indicative of the impact EMDR could have on these diagnoses. An additional consideration is that whereas EMDR has no homework and is typically provided in a onceweekly session, CBT treatment, such as exposure, involves daily homework. Although this produces practice and mastery effects, it also seems to necessitate the spacing of treatment in weekly sessions to accommodate the daily prescribed practice. However, because EMDR does not use daily homework, it is not necessary to engage in a weekly format. What would happen if EMDR treatments occurred in a more concentrated administration in contrast to CBT and exposure therapy protocols?

\section{The Current Study}

Given the paucity of extant research in these areas, this pilot case study investigated the impact of concentrated EMDR treatment on an individual whose presentation met the diagnostic criteria for MDD and panic disorder with agoraphobia, but not for PTSD. It was also designed to evaluate the effectiveness of concentrated EMDR treatment.

\section{Method}

\section{Design}

This study investigated concentrated EMDR treatment using a quantitative case study design, with the understanding that such a design can contribute 
to the literature in several ways. First, a quantitative case design can provide a preliminary investigation of a hypothesis, thus evaluating the potential need for further research (Creswell, 2003). Second, the results of statistical data can inform clinical practice by providing some empirical support to guide more effective treatment (Mertens, 2005). Third, the case study evaluation of a single case can provide in-depth understanding of the individual's phenomenological experience within the boundaries and limitations of said design. This study investigated the outcome of concentrated EMDR treatment for an individual with presenting symptoms of severe depression and anxiety.

\section{The Participant}

To assure the highest level of confidentiality, the individual participant in this study is referred to as "the participant." Appropriate safeguards were placed to assure the protection and ethical treatment of the participant. The participant was a married employed female, in her 30s, and of Euro-American descent. She sought assistance because of significant disruption in her daily functioning. The presenting complaint reemerged when the participant discovered that a family member had reportedly experienced childhood abuse by the same adult male family members who had abused the participant during her own childhood.

\section{EMDR Treatment Schedule}

Treatment followed the established EMDR treatment eight-phase, three-pronged protocol. Three sessions were provided for initial evaluation, and history taking (Phase 1) and preparing the client for treatment (Phase 2). These sessions were provided on a weekly basis. Concentrated EMDR was provided for the reprocessing (Phases 3-8), with twelve 90-minute sessions provided on a three-session per week basis. Two follow-up sessions were provided, at 1-month and 3-month posttreatment. The researcher complied with the EMDR Research Fidelity protocol provided by EMDR International Association (EMDRIA) Research Special Interest Group to assure fidelity.

\section{Assessment}

Both qualitative and quantitative assessments were used to evaluate the presenting complaints. The DSMTR-IV was used to guide the initial qualitative assessment to gather a phenomenological understanding of the participant's experience. In addition to the DSM-TR-IV criteria, a quantitative assessment was performed using the Beck Depression Inventory-II (BDI-II) and Beck Anxiety Inventory (BAI).

The BDI-II (Beck, Steer, Ball, \& Ranieri, 1996) is a 21-item self-report measure assessing severity of symptoms of depression occurring in the last 2 weeks. Each item is scored on a scale value of 0 to 3, with total score ranging from 0 to 63 . Scores from 0 to 13 indicate minimal depression, from 14 to 19 indicate mild depression, from 20 to 28 indicate moderate depression, and from 29 to 63 indicate severe depression. BAI (Beck et al., 1988) is a 21 -item self-report measure assessing severity of symptoms of anxiety occurring in the last week. Each item is scored on a scale value of 0 to 3 , with total score ranging from 0 to 63 . Scores from 0 to 7 indicate minimal anxiety, from 8 to 15 indicate mild anxiety, from 16 to 25 indicate moderate anxiety, and from 26 to 63 indicate severe anxiety.

Assessments were conducted at seven time points: three at baseline (during three history taking and treatment preparatory sessions); one in midtreatment (treatment session 6); one at posttreatment (treatment session 12); and two at follow-up (1 month and 3 months). The BDI-II and BAI were administered on all seven occasions. The diagnostic assessment was conducted only during baseline assessment, although qualitative assessment of the participant's function and status was conducted at all assessment points.

\section{Client History and Presenting Problems}

The participant had a childhood history of sexual and physical abuse by adult male family members over a period of 2 to 3 years, prior to her adolescence stage of development. The participant indicated that related issues had been addressed through traditional talk therapy over a period of 2 years during her later adolescence. She reported no identifiable related symptoms since completing her treatment in adolescence.

The presenting complaint reportedly emerged when the participant discovered that another family member had experienced similar abuse as child by the same adult male family members. Within 1 week of discovering this knowledge, the participant was unable to attend work, perform acts of daily living, had multiple panic attacks daily, lost more than $20 \mathrm{lbs}$, was not sleeping, and had withdrawn from nearly all social interactions.

The participant had been with her spouse for more than 15 years and reported that her partner was understanding and supportive. She described that they had discussed her experiences prior to the development of the presenting complaints. The participant 
related that her marital relationship has been strained from time to time because of ongoing dynamics in the participant's family of origin. The relationship strain had also reemerged with the current presenting complaint.

\section{Pretreatment Assessment}

The participant reported debilitating panic that prevented her from being able to fulfill her work duties, household duties, and many times prevented her from leaving the house. The level of panic and the related functional impairment met the diagnostic criteria for panic disorder with agoraphobia (APA, 2000). The participant also presented with severe lack of interest and pleasure; a loss of more than $20 \mathrm{lbs}$ over the 3 weeks previous to the first meeting; an increased social withdrawal from her spouse, family, and social interactions; suicidal ideation with no plan; and significant lethargy preventing her from performing daily tasks of living. These symptoms satisfied the criteria for severe MDD (APA, 2000). Therefore, the participant presented with a comorbid diagnosis of MDD, severe without psychotic features, and panic disorder with agoraphobia. The disorder of PTSD was ruled out because the participant stated that her current distress was related to her sister's disclosure, and was unrelated to her own childhood sexual abuse; she asserted that she had not had any intrusive memories of her own abuse since her treatment 20 years previously. Therefore, her presentation did not meet diagnostic criteria for PTSD.

These diagnoses were supported by the baseline quantitative assessment using the BDI-II and BAI. The mean baseline score of the BAI equaled 37 and a mean baseline score of the BDI-II equaled 48. By definition, a score of 37 on the BAI indicates "severe anxiety" (Beck, Epstein, Brown, \& Steer, 1988). A score of 48 on the BDI-II indicates "severe depression" (Beck et al., 1996). It should be noted that during the history and assessment sessions, there was a further deterioration in symptoms. (See Figures 1 and 2.)

\section{Course of Treatment}

Phases 1 and 2. Three weekly sessions were provided for Phase 1 (history taking) and Phase 2 (preparation). The participant's history was taken, a diagnostic assessment was completed, and the BDI-II and BAI were administered at the beginning of each session. The "safe place" protocol was administered in the first session designated for Phase 2 and the "container" protocol in the second. The participant and therapist worked together during both sessions to develop the target-sequencing plan for past memories, present triggers, and future desired outcomes.

\section{Case Conceptualization}

The researcher used the AIP model to conceptualize the participant's presenting symptoms. Case conceptualization is required for effective EMDR treatment regardless of the frequency and duration of treatment (Shapiro, 2001). The participant's presenting complaints manifested in cognitive themes of over responsibility, a lack of power, and a sense of worthlessness. The NCs or conclusions of self (Grey, 2010) that structured the treatment were as follows: (a) "I am worthless," (b) "I am trapped," and (c) "It's all my fault." A target-sequencing plan was developed for each NC. In compliance with the AIP/EMDR approach, the participant and researcher agreed to begin with the target-sequencing plan with the earliest memories (Shapiro, 2001). The NC of "I am worthless" was associated with the earliest memories. Further, the theme of worthlessness was pervasive across the participant's self-report. Six memories were listed on the target-sequencing plan A for the NC "I am worthless"; three memories were associated with "I am trapped," listed on target-sequencing plan B; and five memories were associated with "it's all my fault," listed on target sequencing plan C. Each memory was rated by the client using the SUD. All memories were rated with a SUD score of 7 or greater. These targetsequencing plans were used as self-report tools to check for the participant's experiential progress.

The participant also identified present triggers/ experience and future desired outcomes. In addition to past memories on each target-sequencing plan, the participant identified communication with her memories of family, fulfilling her job duties, and general social situation as present triggers. Her future desired outcome were to believe that she was worthwhile, confident, and had energy to participate in her life; to have an internal sense of freedom; and to feel responsible only for herself. The AIP/EMDR approach requires treatment planning to include past, present, and future time frames, the three-pronged approach (Shapiro, 2001).

Phases 3 Through 8. Reprocessing of the identified memories commenced after the three sessions designated for Phases 1 and 2 were completed (session 4), and the EMDRIA research protocol was followed. Processing remained focused on each target memory until the participant reported a SUD score of 0 for that memory. Once the participant reported a SUD score of 0 on the first targeted memory, the researcher and 
participant reevaluated the SUD scores for the remaining targets on target-sequencing plan A. Then, the researcher and participant reprocessed the next memory listed on target-sequencing plan A that had a SUD score greater than 0 . After all of the targeted memories on target-sequencing plan A rated a SUD score of 0 , the researcher and participant continued reprocessing the memories on target-sequencing plan B. The same protocol for reevaluation of targetsequencing plan A was used to reevaluate plans B and C. The EMDR reprocessing sessions lasted for a nonpredetermined length of 12 sessions over 4 weeks to complete all memories associated with the maladaptive themes presented by the participant. No treatment was given after the 12th reprocessing treatment session. No contact occurred between the researcher and the participant between the 12th session, 1-month follow-up, and 3-month follow-up.

Processing of Negative Cognitions: "I'm Worthless" (Target-Sequencing Plan A)

Eight sessions were needed to address the NC "I'm worthless." The memories linked with this NC were regarding childhood medical illnesses, childhood interactions with her sister, and her childhood perception of disappointing her mother. Processing required interweaves related to temporal dual awareness and building ego strength. Processing brought up associated memories related to her childhood friendships and her relationships to her grandmothers. This NC was transformed to a new PC of "I am worthwhile," which the participant endorsed with a high VOC score $($ VOC $=7)$.

\section{Processing of Negative Cognitions:}

\section{"I am Trapped" (Target-Sequencing Plan B)}

Three sessions addressed the NC "I am trapped." The memories linked with this NC were of a childhood nightmare of drowning and being afraid of the dark. Processing required interweaves related to temporal dual awareness. Processing brought up associated memories related to sharing a bedroom with her sister. This NC was transformed to a new PC of "I am free," which the participant endorsed with a high VOC score $($ VOC $=7)$.

Processing of Negative Cognitions: "It's All My Fault" (Target-Sequencing Plan C)

One session addressed the NC "It's all my fault." The memories linked with this NC were regarding her relationships to her mother, sister, husband, and two female friends. Processing did not require interweaves, because this was the final memory and reprocessing session. Processing also did not elicit additional associated memories. This NC was transformed to a new PC of "I am innocent," which the participant endorsed with a high VOC score $(\mathrm{VOC}=7)$.

\section{Present Triggers/Experience and Future Desired Outcomes}

Upon completion of target-sequencing plan A, the researcher and participant reevaluated the initial complaint regarding hearing the news that the participant's sister experienced abuse as a child. Through the reevaluation of target-sequencing plan $\mathrm{A}$, the participant rated the original complaint at a SUD score of 0 . Further, when the PC of "I am worthwhile" was added to the original presenting complaint, the client indicated that the PC was valid, indicated by a VOC score of 7 . Because of these results, the specific present incident of hearing of her sister's abuse was not targeted independently.

Upon completion of target-sequencing plan B, the reevaluation of the original complaints maintained its SUD score of 0 and VOC of 7 . A future template was administered at the completion of target-sequencing plan B, synthesizing the participant's resulting PC of "I am free." The participant envisioned experiencing the PC, positive emotions, and positive body sensations as a template to manage future stresses.

While working through target-sequencing plan C, the participant's marital relationship stresses became elicited during the reprocessing. As each of these stressors arose, it was processed according to the treatment protocols. Upon completion of target-sequencing plan $\mathrm{C}$, reevaluation assessed previously identified triggers in the participant's relationship. During the reevaluation, the participant indicated that the triggers in her current relationships with her spouse and other family members all earned a current SUD score of 0; the PCs of "I am worthwhile," "I am free," and "I am innocent" were all true as indicated by VOC scores of 7 for each. The researcher administered a final future template for each of these PCs to end the final treatment session.

\section{Results}

\section{Changes on Psychometric Measures}

The results of this study are shown in the changes in the scores of the BDI-II and BAI. At baseline, the participant had a mean score of 37 on the BAI (see Figure 1), indicating severe anxiety. The participant had a baseline 


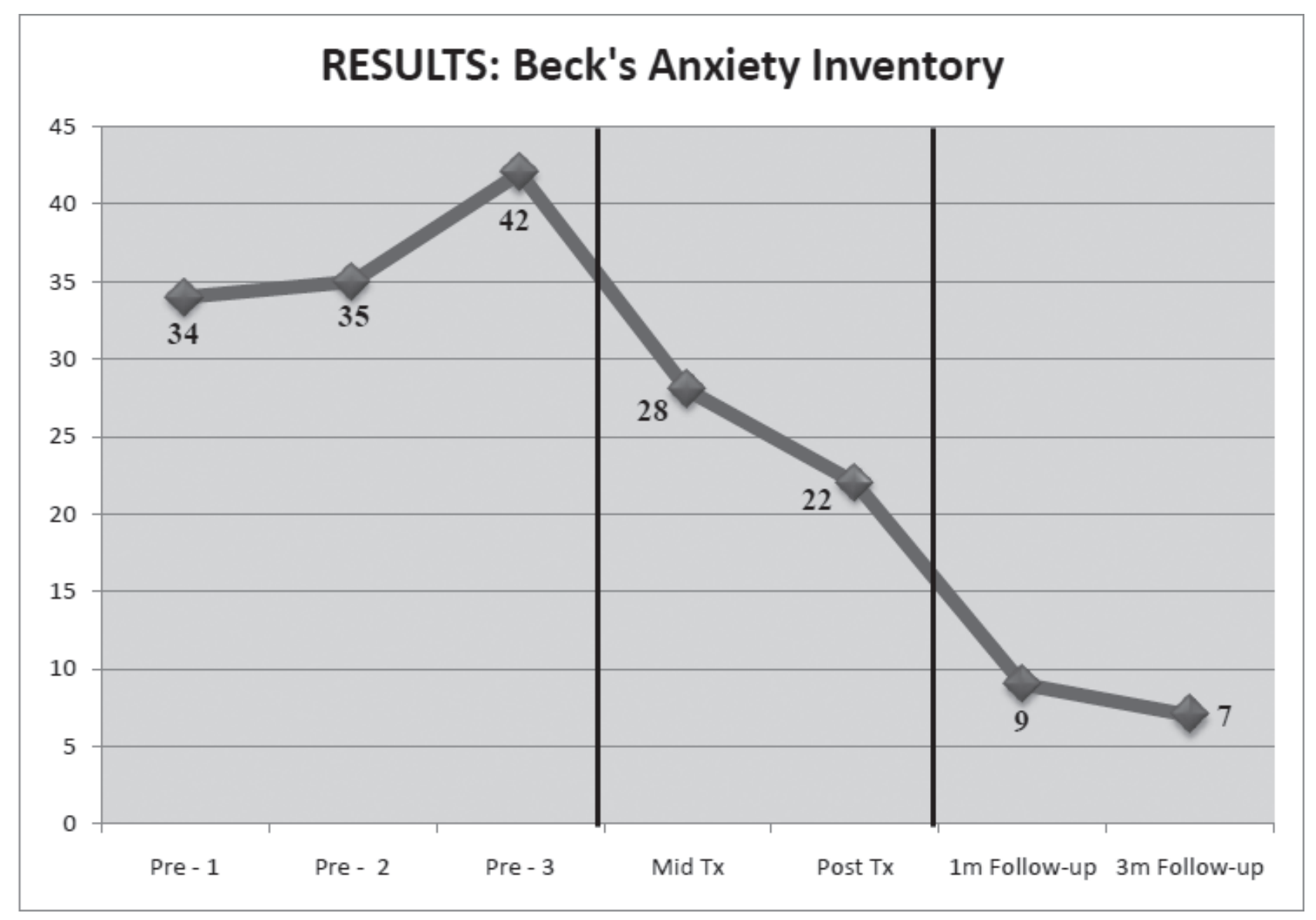

FIGURE 1. BAI: 0-21 indicates very low anxiety; 22-35 indicates moderate anxiety; $36+$ indicates intrusive anxiety.

mean score of 48 on the BDI-II (see Figure 2), indicating severe depression. Midtreatment was defined as reprocessing half of the target-sequencing plan memories to a resolution with a SUD score of 0. Midtreatment occurred on the sixth session of treatment. The sixth session of treatment occurred on the third session of the second week of concentrated treatment (three sessions per week). At midtreatment, the participant scored 28 on the BAI, indicating a decrease in anxiety, although her score was still in the severe range. She scored 30 on the BDI-II indicating a decrease in depression, although her score was still in the severe range.

The final session of treatment was determined when all memories on all target-sequencing plans were resolved to a SUD score of 0 , including present triggers resolved with a SUD score of 0 , and future goals with a VOC score of 7 . Treatment completion occurred on the 12th session, which was the last session of the fourth week of concentrated treatment. During the final session of treatment, posttreatment scores were collected. At posttreatment, the participant scored 22 on the BAI, indicating moderate anxiety, and scored 24 on the BDI-II, indicating moderate depression. It should be noted that the BDI-II collects information during the prior 2 weeks, and consequently, her scores at posttreatment were not fully indicative of her experience on the day of testing but reflected her symptoms over half the course of treatment. Similarly, her BAI scores reflected her symptoms over the prior week. At 1-month followup, the participant scored 9 on the BAI, indicating mild anxiety, and scored 12 on the BDI-II, indicating minimal depression. At 3-month follow-up, the participant scored 7 on the BAI, indicating minimal anxiety, and scored 8 on the BDI-II, indicating normal mood levels (see Figures 1 and 2).

\section{Changes in Function}

By the end of the treatment, the participant reported marked improvements in her functioning. She returned to work and did not require any accommodation to her schedule or job duties. She noted that her energy level had increased, with a corresponding improvement in work performance. She described an improvement in her communication with her husband and indicated that she felt more confident in expressing her thoughts and feelings with him. Her appetite improved and she 


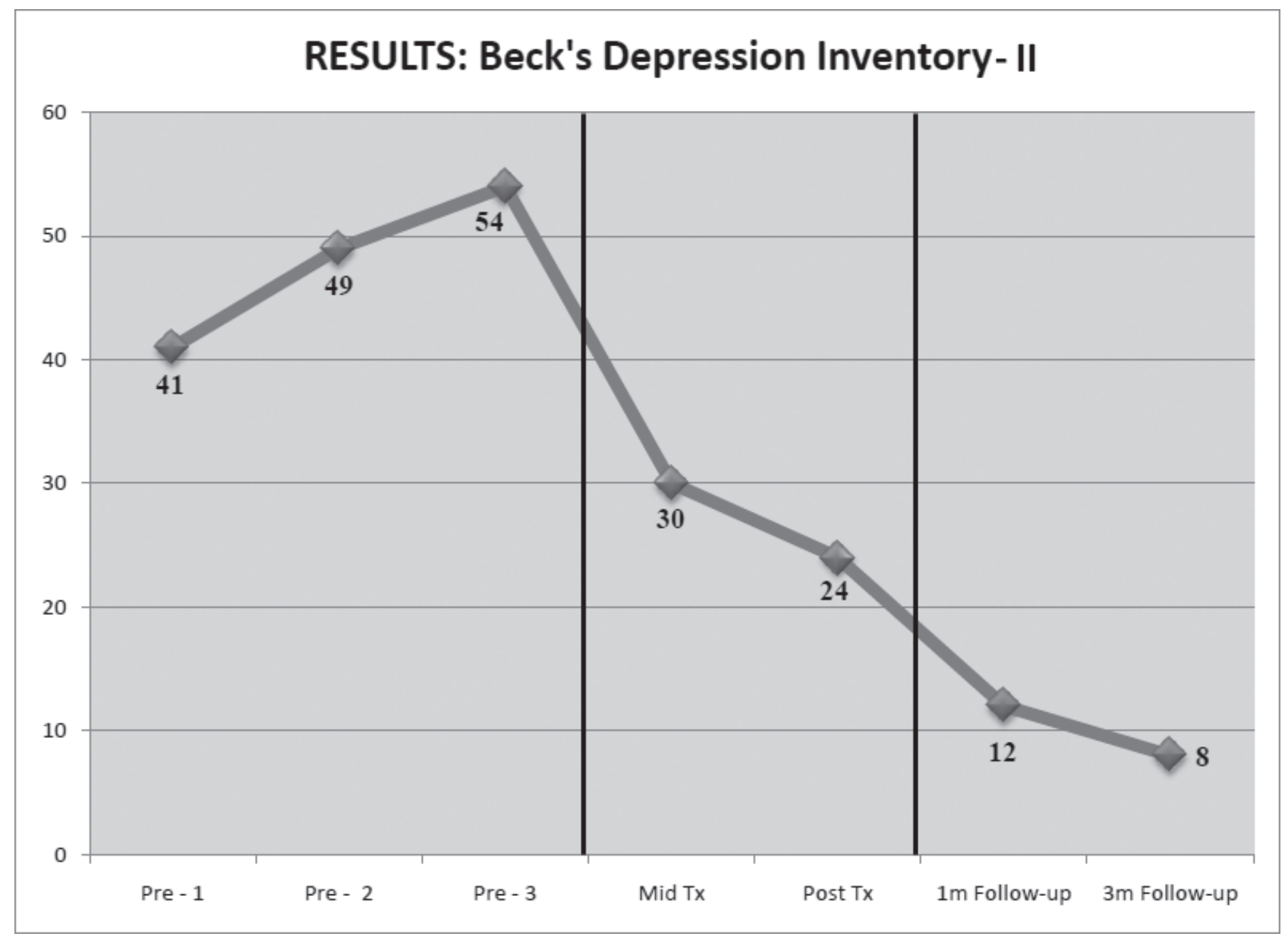

FIGURE 2. BDI-II: 1-10 indicates normal ups and downs; 11-16 indicates mild mood disturbance; 17-20 indicates borderline clinical depression; 21-30 indicates moderate depression; 31-40 indicates severe depression; 40+ indicates extreme depression.

returned to a healthy weight. She further reported that she was more social and was seeking opportunities to expand her circle of friends.

\section{Discussion/Implications}

\section{Limitations}

Limitations of this study include the case study design, which evaluates the experience of one individual. Although the results in this study were profound and clinically significant, they are not generalizable because of the lack of sample size. One cannot rule out the possibility that the results are idiosyncratic and that they can be attributed to the particular features of this one specific individual with her own unique prior history. A second limitation of this study was that the researcher was the treatment provider. Within this study, the dual relationship poses a threat to the validity of the results. The researcher's use of objective measures, a formal treatment fidelity protocol, and his advanced training in EMDR all assisted in decreasing this threat to validity. A third limitation is that the midtreatment administration of the BDI-II measured symptoms experienced during the first 2 weeks of treatment and the posttest administration measured symptoms experienced during the last six sessions of treatment. Consequently, the scores do not reflect the progress at that point in time. Similar problems occurred with the BAI, which collected symptoms during a 1-week (3-session) period. The 1-month and 3-month follow-ups assisted in decreasing this confound.

\section{Concentrated Eye Movement Desensitization and Reprocessing}

The results of this pilot study are noteworthy in indicating that concentrated EMDR may be effective for decreasing emotional suffering in a rapid way. The results found in this study were achieved with 12 EMDR reprocessing sessions within a 1-month. The author argues that the ability to use concentrated in-session treatment illustrates an important difference between EMDR and CBT treatments. 
CBT uses weekly sessions because daily homework completion is needed to ensure that treatment provides maximum benefits. This difference between EMDR and CBT treatments are often obscured in research studies because of the same number of insession treatments given. The author contends that the therapy work done in homework sessions should be considered as additional treatment time for CBT studies. It is recommended that future studies, comparing EMDR to CBT treatments with homework, provide concentrated EMDR to provide a better comparison of the amount of treatment provided to participants. The utility of providing EMDR in a concentrated application was supported in this study. There appeared to be no negative effects from provision of thrice-weekly sessions. These results are consistent with those reported by Wesson and Gould (2009).

Ehlers et al. (2010) investigated intensive cognitive therapy with those suffering from PTSD. Their protocol included 18 hours of treatment in 5-7 working days, homework assignments for the next 5 days, and ongoing homework assignments over the next 3 months. There were also three additional treatment session in between the 1-week and 3-month followup. The results indicate that an intensive cognitive therapy protocol may be effective and are comparable to weekly cognitive treatments on a weekly basis with homework.

Although Ehlers et al. (2010) targeted PTSD and the current study targeted comorbid depression and agoraphobia, some comparisons can be made in favor of concentrated EMDR. In both studies, 18 hours of treatment were administered. Ehlers et al. (2010) eliminated the homework component during the intensive 1-week treatment and then used homework as an additive to the treatment protocol. In the current study, only the 18 hours of the full EMDR protocol were used to support the resolution in the participant's symptoms. Not only was no homework assigned but also no additional treatment sessions occurred between the final session and 3-month follow-up. By comparing the current study to Ehlers et al. (2010), EMDR likely requires less time and resources from a client outside of the actual treatment session and yields comparable results. This comparison further spotlights the dissimilarities between concentrated EMDR and intensive cognitive therapies. These dissimilarities need further research.

The applications of concentrated EMDR highlight the difference in EMDR from other previously compared treatments(i.e., CBT and exposure). Considering the preceding comparison, it is reasonable to suggest that concentrated EMDR may be efficient at decreasing client's cost and time required for efficient symptom resolution. It is possible that concentrated EMDR may be useful and practical in residential and hospital settings, decreasing the length of stay and resources required for stabilization and discharge. Future research is needed to evaluate this possibility and to compare concentrated EMDR with intensive CBT without homework across all levels of care.

\section{EMDR Treatment of Depression and Anxiety}

The results of this study provide insight into the scope of EMDR treatment. Although the efficacy of EMDR treatment for PTSD is established (APA, 2004; Nowill, 2010; VA \& DoD, 2004), its effectiveness for the treatment of other disorders has yet to be adequately investigated (Maxfield, 2009). This pilot study shows how case studies can be used in preliminary investigations.

This study provides preliminary evidence that concentrated EMDR may be effective in treating comorbid depression and panic disorder with agoraphobia. The EMDR treatment promoted an elimination of the participant's diagnoses of panic disorder and MDD by the 3-month follow-up. There was a marked decrease in scores on both the BDI-II and BAI from baseline to 3month follow-up, with follow-up scores indicating no abnormal mood or anxiety symptoms.

The participant reported significant interpersonal challenges at pretreatment. During the treatment, the participant reported being more open to her partner. At the 3-month follow-up, the participant reported marked improvements in the marital relationship in terms of communication and affection. These relational improvements posttreatment may have additional indications for EMDR with couples. It is recommended that further research of concentrated EMDR treatment include measures assessing relationship conflict and quality.

The treatment in this study itself lasted only 1 month with marked decrease in measurement scores, but between the final treatment session and the 1- and 3-month follow-ups, there was no additional treatment. Within the posttreatment/no treatment periods, the participant experienced additional improvements. This improvement may be partly caused by the nature of the Beck instruments. The posttreatment scores reflect anxious and depressive symptoms over the last 1 and 2 weeks of treatment, respectively. Consequently, the 1-month follow-up score may provide a better estimation of treatment outcome. The improvement in symptoms over the follow-up period appears to show a consolidation 
and generalization of treatment effects, with further improvement reported. Improvements at follow-up have been shown in other studies, such as the van der Kolk et al. (2007) randomized clinical trial. Future outcome studies and clinical trials using concentrated EMDR would add support to the results of this study. In addition, longitudinal studies of the lasting effect of concentrated EMDR on depression and anxiety would greatly expand our understanding of the treatment effects of EMDR.

\section{Treatment Implications}

This research study indicates the potential effectiveness of EMDR in resolving disturbing memories, which do not meet diagnostic criteria as traumatic events, but which appear to be experiential contributors to the development of anxiety and depression. In this study, the focus of treatment was on the three negative cognitive themes and the memories of the life events, which had contributed to their formation and maintenance. These included thematic manifestations of worthlessness, powerlessness, and being overly responsible. Reprocessing the memories associated to these cognitive themes with EMDR resulted in an elimination of related distress and a reformulation of the cognitions into adaptive and positive self-referential beliefs. These findings are consistent with those of Bae et al. (2008) and supportive of Shapiro's (2001) theoretical AIP model.

In conclusion, the findings of this pilot study suggest that EMDR treatment may be helpful for individuals suffering from comorbid anxiety and depression. Further, EMDR appears to be effective when applied in a concentrated manner, with three sessions per week. The author suggests that concentrated EMDR could be used in research studies when EMDR is compared to CBT with daily homework, thereby evaluating the differences in treatment application. Further, research studies to compare concentrated EMDR with concentrated CBT using less additive homework would begin to address a more accurate comparison between the treatment approaches.

\section{References}

American Psychiatric Association. (2000). Diagnostic and statistical manual of mental disorders (4th ed., rev.). Washington, DC: Author.

American Psychiatric Association. (2004). Practice guideline for the treatment of patients with acute stress disorder and posttraumatic stress disorder. Arlington, VA: Author.

Bae, H., Kim, D., \& Park, Y. C. (2008). Eye movement desensitization and reprocessing for adolescent depression. Psychiatry Investigation, 5(1), 60-65.
Beck, A. T., Epstein, N., Brown, G., \& Steer, R. A. (1988). An inventory for measuring clinical anxiety: Psychometric properties. Journal of Consulting and Clinical Psychology, 56(6), 893-897.

Beck, A. T., Steer, R. A., Ball, R., \& Ranieri, W. (1996). Comparison of Beck depression inventories -IA and -II in psychiatric outpatients. Journal of Personality Assessment, 67(3), 588-597.

Broad, R. D., \& Wheeler, K. (2006). An adult with childhood medical trauma treated with psychoanalytic psychotherapy and EMDR: A case study. Perspectives in Psychiatric Care, 42(2), 95-105.

Creswell, J. W. (2003). Research design: Qualitative, quantitative, and mixed methods approaches. Thousand Oaks, CA: Sage.

de Jongh, A., \& ten Broeke, E. (2009). EMDR and the anxiety disorders: Exploring the current status. Journal of EMDR Practice and Research, 3(3), 133-140.

Edmond, T., Rubin, A., \& Wambach, K. (1999). The effectiveness of EMDR with adult female survivors of childhood sexual abuse. Social Work Research, 23(2), 103-116.

Ehlers, A., Clark, D. M., Hackmann, A., Grey, N., Liness, S., Wild, J., et al. (2010). Intensive cognitive therapy for PTSD: A feasibility study. Behavioural and Cognitive Psychotherapy, 38(4), 383-398.

Fernandez, I., \& Faretta, E. (2007). Eye movement desensitization and reprocessing in the treatment of panic disorder with agoraphobia. Clinical Case Studies, 6(1), 44-63.

Feske, U., \& Goldstein, A. J. (1997). Eye movement desensitization and reprocessing treatment for panic disorder: A controlled outcome and partial dismantling study. Journal of Consulting and Clinical Psychology, 65(6), 1026-1035.

Goldstein, A. J., de Beurs, E., Chambless, D. L., \& Wilson, K. A. (2000). EMDR for panic disorder with agoraphobia: Comparison with waiting list and credible attention-placebo control conditions. Journal of Consulting \& Clinical Psychology, 68(6), 947-956.

Grey, E. (2010). Use your brain: A neurologically driven application of REBT with children. Journal of Creativity in Mental Health, 5(1), 54-64.

Högberg, G., Pagani, M., Sundin, O., Soares, J., Aberg-Wistedt, A., Tärnell, B., et al. (2007). On treatment with eye movement desensitization and reprocessing of chronic post-traumatic stress disorder in public transportation workers: A randomized controlled study. Nordic Journal of Psychiatry, 61(1), 54-61.

Maxfield, L. (2009). Looking back, moving forward. Journal of EMDR Practice and Research, 3, 210.

Mertens, D. (2005). Research and evaluation in education and psychology (2nd ed.). Thousand Oaks, CA: Sage.

National Institute for Clinical Excellence. (2005). Posttraumatic stress disorder (PTSD): The management of adults and children in primary and secondary care. London: NICE Guidelines.

Nowill, J. (2010). A critical review of the controversy surrounding eye movement desensitization and reprocessing. Counselling Psychology Review, 25(1), 63-70. 
Power, K. G., McGoldrick, T., Brown, K., Buchanan, R., Sharp, D., Swanson, V., \& Karatzias, A. (2002). A controlled comparison of eye movement desensitization and reprocessing versus exposure plus cognitive restructuring, versus waiting list in the treatment of posttraumatic stress disorder. Journal of Clinical Psychology and Psychotherapy, 9, 299-318.

Shapiro, F. (1989). Eye movement desensitization: A new treatment for post-traumatic stress disorder. Journal of Behavior Therapy and Experimental Psychiatry, 20(3), 211-7.

Shapiro, F. (2001). Eye movement desensitization and reprocessing: Basic principles, protocols, and procedures. (2nd ed.). New York: Guilford Press.

Shapiro, E., \& Laub, B. (2008). Early EMDR intervention (EEI): A summary, a theoretical model, and the recent traumatic episode protocol (R-TEP). Journal of EMDR Practice and Research, 2(2), 79-96.

Solomon, R. M., \& Shapiro, F. (2008). EMDR and the adaptive information processing model: Potential mechanisms of change. Journal of EMDR Practice and Research, 2(4), 315-325.

Stewart-Grey, E. (2008). De-stress: A qualitative investigation of EMDR treatment. Available from ProQuest Dissertations \& Theses: Full Text (UMI No. 3329984)
Uribe, M. E. R., Ramírez, E. O. L., \& Mena, I. J. (2010). Effect of the EMDR psychotherapeutic approach on emotional cognitive processing in patients with depression. The Spanish Journal of Psychology, 13(1), 396-405.

U.S. Department of Veterans Affairs, \& U.S. Department of Defense. (2004). VA/DoD clinical practice guideline for the management of post-traumatic stress. Washington, DC: Author.

van der Kolk, B. A., Spinazzola, J., Blaustein, M. E., Hopper, J. W., Hopper, E. K., Korn, D. L., et al. (2007). A randomized clinical trial of eye movement desensitization and reprocessing (EMDR), fluoxetine, and pill placebo in the treatment of posttraumatic stress disorder: Treatment effects and long-term maintenance. Journal of Clinical Psychiatry, 68(1), 37-46.

Wesson, M., \& Gould, M. (2009). Intervening early with EMDR on military operations. Journal of EMDR Practice and Research, 3(2), 91-97.

Wolpe, J. (1990). The practice of behavior therapy (4th ed.). New York: Pergamon Press.

Correspondence regarding this article should be directed to Earl Grey, 875 Greentree Rd, Seven Parkway Center, Suite 240, Pittsburgh, PA 15220. E-mail: drearlgrey@ gmail.com 